\title{
Gamma-ray Flares from the Gravitationally Lensed Blazar B0218+357
}

\section{S. Buson*}

Center for Research and Exploration in Space Science and Technology (CRESST)

NASA Goddard Space Flight Center, Greenbelt, MD 20771, USA

University of Maryland Baltimore County, Baltimore, MD 21250, USA

E-mail: sara.buson@nasa.gov

\section{C. Cheung}

Space Science Division, Naval Research Laboratory, Washington, DC 20375-5352, USA

E-mail: Teddy.Cheung@nrl.navy.mil

\section{S. Larsson}

Department of Physics, Stockholm University, AlbaNova, SE-106 91 Stockholm, Sweden The Oskar Klein Centre for Cosmoparticle Physics, AlbaNova, SE-106 91 Stockholm, Sweden Department of Astronomy, Stockholm University, SE-106 91 Stockholm, Sweden

\section{J. D. Scargle}

Space Sciences Division, NASA Ames Research Center, Moffett Field, CA 94035-1000, USA

\section{J. Finke}

Space Science Division, Naval Research Laboratory, Washington, DC 20375-5352, USA

\section{on behalf of the Fermi-LAT Collaboration}

The double-image gravitationally lensed blazar B0218+357 displayed several intervals of enhanced activity at $\gamma$-rays. Fermi-LAT observations based on the 2012 flaring interval led to the measurement at $>100 \mathrm{MeV}$ energies of a delay between the two lensed images of $11.46 \pm 0.16$ days. The delay is about 1 day longer than previously measured at radio wavelengths. Renewed flaring activity has been detected recently in 2014 when the source displayed an exceptionally hard spectrum, significantly different from the 2012 interval, and a different behavior in the second image. We present new Fermi-LAT variability and spectral studies of the system and discuss them in the context of previous measurements.

The 34th International Cosmic Ray Conference,

30 July- 6 August, 2015

The Hague, The Netherlands

\footnotetext{
* Speaker.
} 


\section{Introduction}

B0218+357 is a blazar at $z=0.944 \pm 0.002$ [1] lensed by a galaxy at redshift $z=0.6847$ [2]. In the radio band the system is characterized by two images separated by 335 milli-arcseconds, with a brighter western image "A" leading the fainter eastern "B" image and an Einstein ring [3, 4]. A flux ratio $(\mathrm{A} / \mathrm{B})$ of $\sim 3$ or 4 was reported from high-frequency $(\sim 15 \mathrm{GHz})$ radio observations. These are the most representative intrinsic radio values because at these frequencies propagation effects are minimal (i.e. they are absorption free) [5]. It is worth mentioning that optical and infrared observations find the B image being brighter than the A image; this is likely attributed to a combination of extinction of the A image and microlensing [6, 7].

Several different values were provided also for the delay between the two images using the radio dataset. [8] and [9] inferred a delay of $\Delta t_{\mathrm{r}}=10.5 \pm 0.2$ and $10.1 \pm 0.8$ days, respectively, using different dataset. Subsequent timing analysis of the same radio lightcurve of [9] by [10] revealed two other possible delay values, $\Delta t_{\mathrm{r}}=9.9_{-0.9}^{+4.0}$ or $11.8 \pm 2.3$ days.

Although it has been studied in detail for long time, B0218+357 attracted renewed interest since when it was detected for the first time in $\gamma$-rays by the Fermi Large Area Telescope [LAT; 11]. Over the initial 4-years of LAT observations, B0218+357 displayed a $\gamma$-ray flux of $F_{E>100 \mathrm{MeV}}=$ $(2.4 \pm 0.9) \times 10^{-8}$ photons $\mathrm{cm}^{-2} \mathrm{~s}^{-1}$ and a photon index of $\Gamma=2.28 \pm 0.03$ [3FGL J0221.1+3556; third Fermi-LAT Catalog, 3FGL, 12].

The Fermi-LAT has observed two major episode of $\gamma$-ray flaring activity from this blazar. While previously no significant change in the $\gamma$-ray spectrum has been reported through the flaring phase in late-2012, during the most recent enhanced state in July 2014 the source displayed an exceptionally hard spectrum. The latter led to the detection of very high energy (VHE, $E>0.1$ $\mathrm{TeV}) \boldsymbol{\gamma}$-rays from B0218+357 by the MAGIC telescopes, establishing this source as the most distant VHE emitter known to date $[13,14]$. In the following we present the characterization of the major $\gamma$-ray flaring phases displayed by this object.

\section{Gamma-ray flares from $\mathrm{B} 0218+357$}

\subsection{Enhanced Phase during Late 2012}

The first prominent outburst activity observed by Fermi-LAT was a long-lasting one characterized by a series of strong subsequent flares that developed between 2012 June and subsided by 2013 March. The bulk of the $\gamma$-ray emission persisted for about four months and provided a rich dataset to study the lensing properties of the system. Applying an auto-correlation function (ACF) to the evenly sampled 6-hr binned lightcurve focused on the 2012-2013 enhanced $\gamma$-ray activity, [15] found a single dominant correlation peak whose significance was evaluated to be $9 \sigma$. The delay estimate inferred with this $\gamma$-ray data was $11.46 \pm 0.16$ days $(1 \sigma)$, and represents the first robust $\gamma$-ray lensing delay measurement obtained with $\gamma$-rays.The LAT delay measurement uncertainty is competitive with the best previous radio measurement. Importantly, during this period no indication for spectral variability was found by the previous studies. A single power-law fit to the source spectrum over the 1-day binned lightcurve points showed an average $\gamma$-ray photon index $\Gamma=2.31 \pm 0.02$, consistent with the value derived before the start of the flaring as well as consistent with its 3 FGL value. 


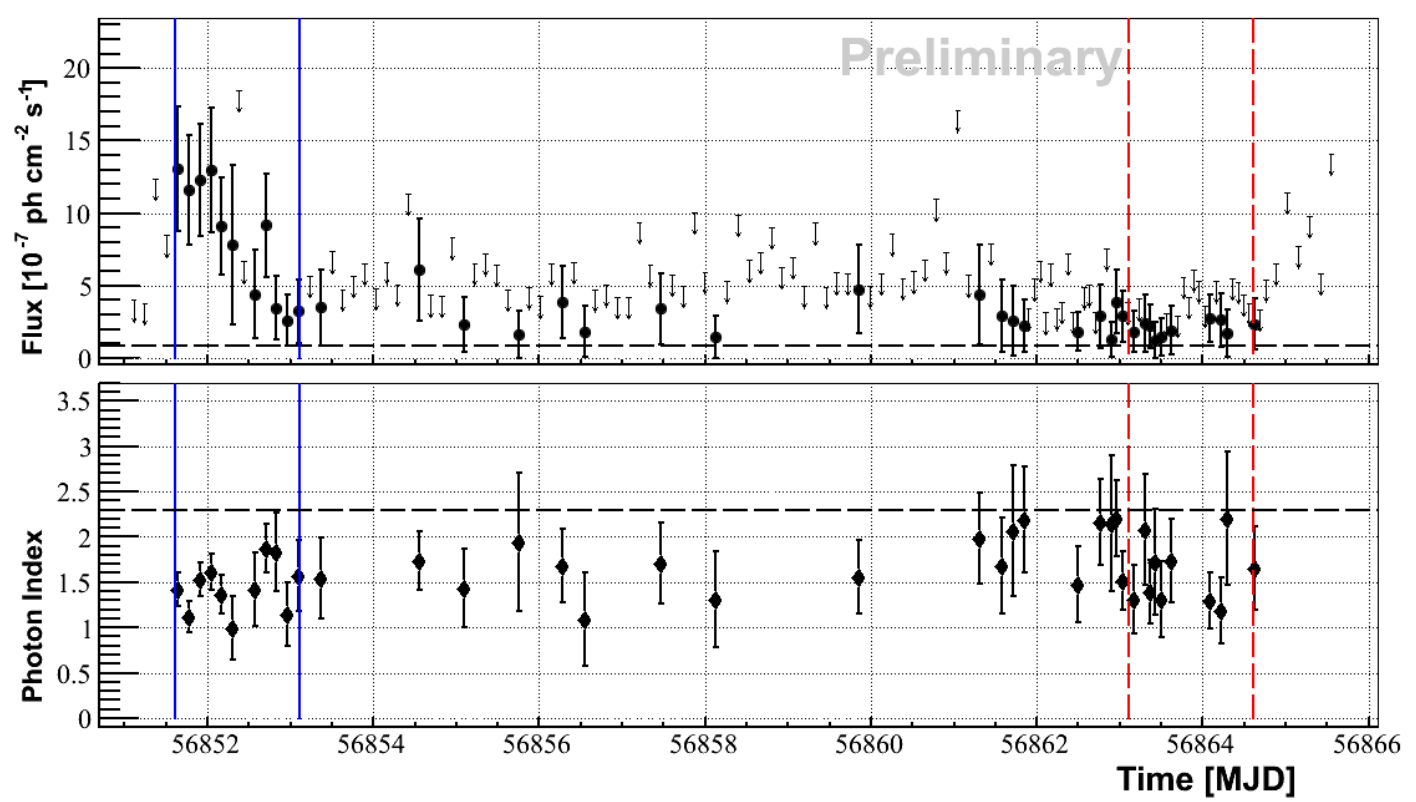

Figure 1: Fermi-LAT lightcurve computed in 1.6-hr orbit bins at energies $E>100 \mathrm{MeV}$ (upper panel) and the corresponding photon indices (lower panel). Vertical lines indicate the intervals of the flare (solid blue) and corresponding delayed emission (dashed red) and are offset by 11.5 days. Filled points represent $>2 \sigma$ detections while downward arrows are upper limits. Horizontal dashed lines indicate the 3FGL average values.

\subsection{Renewed activity in $\mathbf{2 0 1 4}$}

B0218+357 underwent a second flaring phase during 2014 July [16, 17]. The enhanced activity in this occasion was restricted to less than 2 weeks. The bulk of the $\gamma$-ray emission was observed between 2014 July 13 and 27 (MJD 56851-56865) and remarkably characterized by a distinctive hard spectrum. Based on a preliminary analysis, on 2014 July 13 and 14 (MJD 56851 and MJD 56852) the source was observed with respective daily averaged fluxes $(E>100 \mathrm{MeV}$ ) of $(6.5 \pm 1.4) \times 10^{-7}$ photons $\mathrm{cm}^{-2} \mathrm{~s}^{-1}$ with $\Gamma=1.4 \pm 0.1$ and $(6.7 \pm 1.5) \times 10^{-7}$ photons cm $^{-2}$ $\mathrm{s}^{-1}$ with $\Gamma=1.6 \pm 0.1$. Because the gamma-ray delay was previously measured, LAT Target of Opportunity observations were triggered to trace the expected delayed emission, about 11 days after. B0218+357 was detected again by the Fermi-LAT through 2014 July 24 to 26. Noteworthy, emission at VHE was subsequently reported by the MAGIC telescopes in coincidence with the expected delayed $\gamma$-ray flare $[13,14]$. In the following we present the preliminary analysis of the $2014 \gamma$-ray dataset.

\section{Remarkably hard spectral phase during 2014}

\subsection{Fermi-LAT analysis}

LAT data were extracted from a circular region of interest (ROI) of $15^{\circ}$ radius centered at the B0218 +357 radio position, R.A. $=35^{\circ} .27279$, Decl. $=35^{\circ} .93715[\mathrm{~J} 2000 ; 18]$ and analyzed in 
the energy range $0.1-300 \mathrm{GeV}$ using the standard Fermi Science Tools (version v9r34p1) in combination with the P7REP_SOURCE_V15 LAT Instrument Response Functions. We applied the gtmktime filter (\#3) to the LAT data following the FSSC recommendations ${ }^{1}$. According to this prescription, time intervals when the LAT boresight was rocked with respect to the local zenith by more than $52^{\circ}$ and events with zenith angle $>100^{\circ}$ were excluded to limit the contamination from Earth limb photons. The spectral model of the region included all sources located within the ROI with the spectral shapes and the initial parameters for the modeling set to those reported in the 3FGL [12] as well as the isotropic (iso_source_v05.txt) and Galactic diffuse (gll_iem_v05.fit) components.

Following the approach of [15] we computed the LAT lightcurve with 1.6-hr orbit binning shown in Fig. 1 for the interval of interest. A long-term lightcurve was also produced to look for other flaring activity but only the 2014 July flares were evident. When fitting the lightcurve bins, B0218+357 was modeled with a single power-law with photon index fixed to the average found throughout the studied interval (upper panel of Fig. 1). Subsequently, to characterize the photon index variations and search for spectral changes, we also repeated the same analysis with the index free to vary (bottom panel of Fig. 1). In the analysis, 95\% confidence level upper limits were computed when the significance of the detection was below $2 \sigma$. To assess the significance we used the test statistic ${ }^{2}$ (TS) as described in [19].

\subsection{Light Curve}

Our preliminary study of data between MJD 56850-56865 confirms the detection of B0218+357 in enhanced state. Figure 1 presents the 1.6-hr orbit lightcurve $(>100 \mathrm{MeV})$ and the corresponding photon index versus time variations during this time interval. A first flaring phase, corresponding to the emission from image A, is well defined by a fast flux increase characterized by an exceptional hard spectrum. This phase is identified by several consecutive detections with significance $>3 \sigma$ happening within $\sim 1.5$ days, as denoted in Figure 1 by the two continuous vertical lines between MJD 56851.608-56853. During this interval the photon index is found to be on average significantly smaller (below 2) with respect to the value of 2.3 reported in previous LAT studies [15], displaying in several bins values close to 1.5 . Noteworthy, the two highest energy photons positionally consistent with the source are a $95 \mathrm{GeV}$ and a $54 \mathrm{GeV}$ event, detected at $\sim \mathrm{MJD} 56852$, i.e. at the time of the initial flare. Immediately after this short outburst the source returned to quiescence, displaying only sporadic activity for the subsequent $\sim 11$ days.

Then, at the time of the expected delayed emission, the source is detected again as denoted by a dense series of bins around MJD 56863. These detections happen $\sim 11.5$ days later. The series of significant consecutive intervals are distinguished again by a small photon index $(\Gamma<2)$. Based on the hard-spectrum signature we argue that this second flaring phase corresponds to the emission from image B. The two red dashed vertical lines in Fig. 1, at MJD 56863.068-56864.46, denote the $<2$-day interval of flare $A$, shifted by 11.5 days, i.e. the previously measured $\gamma$-ray delay. Interestingly, it can be noted that the B-image flare appears to not perfectly match the pattern of

\footnotetext{
${ }^{1}$ http://fermi.gsfc.nasa.gov/ssc/data/analysis/documentation/Cicerone/Cicerone_Likelihood/Exposure.html

${ }^{2}$ The TS corresponds roughly to the square of the significance assuming one degree of freedom.
} 
flare A and is preceded by some additional flaring activity prior to the expected 11.5 day delayed emission.

Formally, the ACF computed on the LAT lightcurve of 1-day binning between MJD 5684956865 indicates a peak around 11 days (not shown). Though its statistical significance has not yet been estimated it is notable that the peak is broadly consistent with the previous $\gamma$-ray delay of $11.46 \pm 0.16$ days.

We compared the flux measured during the first $\sim 1.5$-day flaring episode, beginning at MJD 56851.625 , with the observation shifted by 11.5 days. We derive the resultant average flux ratio A/B $\sim 4$ which is close to what observed in the radio band ( $\sim 3$ or 4 ) but interestingly stands apart from the previous $\gamma$-ray estimate $(\sim 1)$. Note that [20] did not find evidence for the delayed emission applying the approach of [21] to the 2014 July $\gamma$-ray data and explained it as due to microlensing.

\section{Summary}

Previous analysis of LAT data for the 2012 flares of the gravitationally lensed blazar B0218+357 found a $\gamma$-ray delay measurement of $11.46 \pm 0.16$ days and a flux ratio estimate between images (A/B) consistent with $\sim 1$. From a preliminary analysis of LAT data for new flaring detected in 2014 July, we found that the latter flares are characterized by peculiar hard spectra, with photon index $\Gamma<2$. A series of significant detections with hard spectral signature is observed about 11.5 days after flare A. We identify this with the emission from the delayed image B. The average flux ratio (A/B) observed over the 2014 flaring episode is $\sim 4$ : while this factor is different with respect to the previous $\gamma$-ray measurement, it is closer to the radio observations ( $\sim 3$ or 4$)$. Recently [20] suggested that the variability of the $\gamma$-ray magnification factor could be due to microlensing. A 95 $\mathrm{GeV}$ and a $54 \mathrm{GeV}$ photon were detected by the LAT during the 2014 first flare (A image) but were not observed during the second flare of image B. Nevertheless, the MAGIC collaboration reported the significant $(>5 \sigma)$ detection of B0218+357 about 11.5 days after flare A. This ensures that the spectrum of the second image also extends up to $\sim 0.1 \mathrm{TeV}$.

\section{Acknowledgments}

The Fermi-LAT Collaboration acknowledges support for LAT development, operation and data analysis from NASA and DOE (United States), CEA/Irfu and IN2P3/CNRS (France), ASI and INFN (Italy), MEXT, KEK, and JAXA (Japan), and the K.A. Wallenberg Foundation, the Swedish Research Council and the National Space Board (Sweden). Science analysis support in the operations phase from INAF (Italy) and CNES (France) is also gratefully acknowledged. Work by C.C.C. was supported at NRL by NASA DPR S-15633-Y and Guest Investigator program 13FERMI13-0009. S.L. acknowledges support by a grant from the Royal Swedish Academy Crafoord Foundation.

\section{References}

[1] J. G. Cohen, C. R. Lawrence, and R. D. Blandford, The Redshift of the Lensed Object in the Einstein Ring B0218+357, Astrophys. J. 583 (Jan., 2003) 67-69, [astro-ph/ 0209457 ]. 
[2] I. W. A. Browne, A. R. Patnaik, D. Walsh, and P. N. Wilkinson, The Redshift of the Lensing Galaxy in the Gravitationally Lensed System B:0218+35.7, Mon. Not. R. Astron. Soc. 263 (Aug., 1993) L32.

[3] C. P. O’Dea, S. A. Baum, C. Stanghellini, A. Dey, W. van Breugel, S. Deustua, and E. P. Smith, Radio and optical observations of 0218+357 - The smallest Einstein ring?, Astron. J. 104 (Oct., 1992) 1320-1330.

[4] A. R. Patnaik, I. W. A. Browne, L. J. King, T. W. B. Muxlow, D. Walsh, and P. N. Wilkinson, B0218+35.7 - A gravitationally lensed system with the smallest separation, Mon. Not. R. Astron. Soc. 261 (Mar., 1993) 435-444.

[5] R. Mittal, R. Porcas, and O. Wucknitz, Free-free absorption in the gravitational lens JVAS B0218+357, Astron. Astrophys. 465 (Apr., 2007) 405-415, [astro-ph/ 0607623 ].

[6] E. E. Falco, C. D. Impey, C. S. Kochanek, J. Lehár, B. A. McLeod, H.-W. Rix, C. R. Keeton, J. A. Muñoz, and C. Y. Peng, Dust and Extinction Curves in Galaxies with z>0: The Interstellar Medium of Gravitational Lens Galaxies, Astrophys. J. 523 (Oct., 1999) 617-632, [astro-ph/9901037].

[7] N. Jackson, E. Xanthopoulos, and I. W. A. Browne, NICMOS images of JVAS/CLASS gravitational lens systems, Mon. Not. R. Astron. Soc. 311 (Jan., 2000) 389-396, [astro-ph/9909474].

[8] A. D. Biggs, I. W. A. Browne, P. Helbig, L. V. E. Koopmans, P. N. Wilkinson, and R. A. Perley, Time delay for the gravitational lens system B0218+357, Mon. Not. R. Astron. Soc. 304 (Apr., 1999) 349-358, [astro-ph/9811282].

[9] A. S. Cohen, J. N. Hewitt, C. B. Moore, and D. B. Haarsma, Further Investigation of the Time Delay, Magnification Ratios, and Variability in the Gravitational Lens 0218+357, Astrophys. J. 545 (Dec., 2000) 578-590, [astro-ph/ 001004 9].

[10] E. Eulaers and P. Magain, Time delays for eleven gravitationally lensed quasars revisited, Astron. Astrophys. 536 (Dec., 2011) A44, [arXiv:1112.2609].

[11] W. B. Atwood et al., The Large Area Telescope on the Fermi Gamma-Ray Space Telescope Mission, Astrophys. J. 697 (June, 2009) 1071-1102, [arXiv: 0902.1089 ].

[12] Acero F. et al., Fermi Large Area Telescope Third Source Catalog, Astrophys. J. Suppl. Ser. 218 (June, 2015) 23, [arXiv:1501.02003].

[13] R. Mirzoyan, Discovery of Very High Energy Gamma-Ray Emission From Gravitationally Lensed Blazar S3 0218+357 With the MAGIC Telescopes, The Astronomer's Telegram 6349 (July, 2014) 1.

[14] Sitarek J. et al., Detection of very-high-energy gamma rays from the most distant and gravitationally lensed blazar QSO B0218+357 using the MAGIC telescope system, in this proceedings, 2015. 
[15] C. C. Cheung et al., Fermi Large Area Telescope Detection of Gravitational Lens Delayed $\gamma$-Ray Flares from Blazar B0218+357, Astrophys. J. Lett. 782 (Feb., 2014) L14, [arXiv:1401.0548].

[16] S. Buson and C. C. Cheung, Fermi LAT Detection of a Hard Spectrum Gamma-ray Flare from Gravitationally Lensed Blazar S3 0218+357, The Astronomer's Telegram 6316 (July, 2014) 1.

[17] S. Buson, C. C. Cheung, S. Larsson, and J. D. Scargle, Fermi-LAT Detection of a Hard Spectrum Flare from the Gravitationally Lensed Blazar B0218+357, arXiv:1502.03134 (Feb., 2015) [arXiv:1502.0313].

[18] A. R. Patnaik, I. W. A. Browne, P. N. Wilkinson, and J. M. Wrobel, Interferometer phase calibration sources. I - The region 35-75 deg, Mon. Not. R. Astron. Soc. 254 (Feb., 1992) 655-676.

[19] J. R. Mattox, D. L. Bertsch, J. Chiang, B. L. Dingus, S. W. Digel, J. A. Esposito, J. M. Fierro, R. C. Hartman, and others, The Likelihood Analysis of EGRET Data, Astrophys. J. 461 (Apr., 1996) 396-+.

[20] I. Vovk and A. Neronov, Microlensing constraint on the size of the gamma-ray emission region in blazar B0218+357, arXiv (July, 2015) [arXiv: 1507.01092].

[21] A. Neronov, I. Vovk, and D. Malyshev, Central engine of a gamma-ray blazar resolved through the magnifying glass of gravitational microlensing, Nature Physics (July, 2015) doi:10.1038/nphys3376. 